\title{
APLICACIÓN DE MODELOS PARA LA GENERACIÓN DE LA ISOTERMA DE ADSORCIÓN DE METANO EN UNA MUESTRA DE SHALE Y SU IMPACTO EN EL CÁLCULO DE RESERVAS
}

Cristian Alexis Murillo Martínez ${ }^{1 *}$, Ovidio Andrez Gómez Rodríguez², Olga Patricia Ortiz Cancino ${ }^{3}$ Samuel Fernando Muñoz Navarro ${ }^{4}$

*A quien debe dirigirse la correspondencia

\section{RESUMEN}

El comportamiento de diferentes modelos de isotermas de adsorción aplicados a los datos experimentales obtenidos por Gasparik et al. (2012) en una prueba de adsorción de metano, fue modelado y analizado, con el fin de determinar el que mejor representa los datos experimentales. Entre los modelos utilizados se encuentran algunos de dos parámetros como Langmuir, Temkin y Freundlich, otros de tres parámetros como Langmuir, DubininRadushkevich, Toth y Sips; de cuatro parámetros como Dubinin-Astakhov y Fritz-Schlunder y finalmente el modelo de cinco parámetros propuesto por Fritz-Schlunder. Según los resultados obtenidos, los modelos presentaron mejor ajuste a medida que empleaban mayor número de parámetros en sus ecuaciones.

Posteriormente se calculó la capacidad de almacenamiento total de gas para una formación hipotética con algunos datos de la formación Posidonea empleando el método propuesto por Ambrose et al (2010), el cual se modificó reemplazando el término que tiene en cuenta el gas adsorbido con los modelos mencionados anteriormente, a fin de determinar su influencia en las estimaciones de volumen de gas libre y de gas adsorbido. Con los modelos que presentaron un menor porcentaje de error, se obtuvieron datos más conservadores en el cálculo del gas adsorbido y de gas total, lo cual indica la importancia de la adecuada selección del modelo para el cálculo de reservas, dado que conlleva alto impacto técnico y económico.

Palabras clave: Shale, gas, yacimiento, isotermas, adsorción.

\section{APPLICATION OF ADSORPTION ISOTHERM MODELS OF METHANE ON A SHALE SAMPLE AND THEIR IMPACT ON RESERVES ESTIMATION}

\begin{abstract}
Several adsorption isotherm models were analyzed for the purpose of fitting the experimental data published by Gasparik et al. (2012) obtained in a methane adsorption experiment on a shale sample from the Posidonea formation in The Netherlands. The following are the ten models used in this study; some of them consist of two parameters such as Langmuir, Temkin and Freundlich, the three parameter models: Langmuir, DubininRadushkevich, Toth and Sips, four parameters: Dubinin-Astakhov and Fritz-Schlunder and the five-parameter Fritz Schlunder correlation. The results show a better fit to the experimental data for those models with the highest number of parameters.
\end{abstract}

1. Escuela de Ingeniería de Petróleos, Universidad Industrial de Santander, Bucaramanga, Colombia. cristian. murillo1@correo.uis.edu.co

2. Escuela de Ingeniería de Petróleos, Universidad Industrial de Santander, Bucaramanga, Colombia. ovidio. gomez@correo.uis.edu.co

3. Escuela de Ingeniería de Petróleos, Universidad Industrial de Santander, Bucaramanga, Colombia. oportizc@ uis.edu.co

4. Escuela de Ingeniería de Petróleos, Universidad Industrial de Santander, Bucaramanga, Colombia. samuel@ uis.edu.co 
Subsequently, The total gas storage capacity in a hypothetical formation was calculated using some data of the Posidonea formation and the methodology proposed by Ambrose et al (2010), which was modified afterwards by substituting the term taking into account the adsorbed gas for the models mentioned earlier in this article in order to determine their influence in the free and adsorbed gas calculations. In this way, the models with the lowest Average Percentage Error (APE) generally calculated a lower volume of adsorbed and total gas compared to those models with the highest APE, which indicates the importance of a proper model selection for calculating reserves, since it implies high technical and economic impact.

Keywords: Shale, gas, reservoir, isotherms, adsorption.

\section{INTRODUCCIÓN}

El gas en los yacimientos tipo shale se almacena de tres formas diferentes: Como gas libre que se encuentra en los microporos y fracturas, como gas adsorbido en la superficie de los minerales y el kerógeno, y como gas disuelto en la materia orgánica, siendo este último despreciable[6]. En consecuencia, el contenido total de gas en shales es considerado como la sumatoria del gas adsorbido y el gas libre; en donde el gas adsorbido representa de un 20 a un $80 \%$ de las reservas totales y de las tasas de recuperación, además de mostrar una fuerte influencia en la producción a etapas tardías, y prolongar la vida productiva del yacimiento [11]. Por esta razón, es necesario entender el fenómeno de adsorción el cual es un criterio que gobierna el cálculo de las reservas y las tasas producción de gas en shales.

Las isotermas de adsorción se desarrollaron para modelar el comportamiento del gas adsorbido a temperatura constante como función de la presión o la concentración de gas, con la finalidad de generar una tendencia e interpolar o extrapolarla a condiciones en las cuales no es posible realizar las mediciones por razones técnicas o económicas.

Existe una vasta cantidad de modelos en la literatura que son empleados con dicho propósito; en este estudio se analizaron modelos bastante conocidos de 2, 3, 4 y 5 parámetros para apreciar los beneficios al aplicar cada uno de ellos a una prueba de adsorción de metano en una muestra de shale.

\section{MODELOS DE ISOTERMAS DE ADSORCIÓN}

\subsection{Modelos de Dos PARÁMETROS}

\subsubsection{Modelo de FreundLich}

Este modelo se fundamenta en la adsorción multicapa en una superficie heterogénea con energía uniforme [2] y se considera como la primera y mejor ecuación de una isoterma empírica, la cual está dada por:
Vads $=(k P)^{1 / m}$

Con $m>1$.

$V_{a d s}$ es el volumen de gas adsorbido en pies cúbicos estándar [pce] por tonelada de adsorbente [ton], $P$ es la presión en [psi], la constante $k$ es un indicador aproximado de la capacidad de adsorción, mientras que $1 / m$ es función de la fuerza del proceso de adsorción. Si $1 / m$ está por debajo de uno, indica un proceso de adsorción normal. Las constantes $k$ y $m$ cambian con un incremento en la temperatura para reflejar que la cantidad adsorbida aumenta de una forma más lenta y se requieren presiones más altas para saturar la superficie de adsorción [12].

El modelo de Freundlich funciona adecuadamente en un rango medio de presiones; sin embargo falla a altas presiones y bajas temperaturas debido a que la isoterma no propone un valor límite de $V_{a d s}$ cuando la presión tiende a infinito [12].

\subsubsection{MOdelo de LANGMUIR}

Según Clarkson et al. (1997) [4], la relación empírica de Langmuir se describe como:

$$
\text { Vads }=V_{L} \frac{(b P)}{1+(b P)}
$$

Donde:

$V_{\text {ads }}$ es la cantidad de sustancia adsorbida en pies cúbicos estándar por tonelada de adsorbente (Roca), $V_{L}$ representa la capacidad máxima de adsorción de gas [pce/ton], $b$ es la afinidad de adsorción (Constante empírica) y $p$ la presión [psi]. Además, un valor importante en la isoterma de Langmuir es la presión a la cual se ha adsorbido la mitad de la capacidad máxima de adsorción $V_{L}$, y se denomina la presión de Langmuir $\mathrm{P}_{L}$.

El modelo de Langmuir asume que existe un estado de equilibrio dinámico (a temperatura y presión constantes) entre las especies adsorbidas y no adsorbidas, y que la 
adsorción se restringe a una sola capa (Quimisorción). Además, supone que la superficie adsorbente es homogénea energéticamente.

\subsubsection{Modelo de Temkin}

Este modelo considera las interacciones de la sustancia adsorbida con la superficie de adsorción, y sugiere que debido a esas interacciones el calor de todas las moléculas en la capa de adsorción debería disminuir linealmente con la cobertura [14]. La isoterma de Temkin se expresa en la forma siguiente:

$$
\text { Vads }=\frac{R T}{f}(\ln A \tau P)
$$

Donde $R$ es la constante de los gases, $T$ es la temperatura, $f$ es la constante de la isoterma de Temkin y A $\tau$ es la constante de unión de equilibrio.

Si:

$$
C=\frac{R T}{f}
$$

Entonces la ecuación de Temkin toma la forma:

$$
\text { Vads }=C * \ln (A \tau P)
$$

\subsection{MODELOS DE TRES PARÁMETROS}

\subsubsection{MOdelo de LANGMUIR}

Según Gasparik et al. (2012) [7], este modelo modificado de Langmuir emplea tres parámetros para generar mayor exactitud en sus resultados. Dicha ecuación se presenta a continuación:

$$
\text { Vads }=V_{L} \frac{p}{p+p_{L}}\left(1-\frac{\rho_{g}(p, T)}{\rho_{a d s}}\right)
$$

Donde:

$\rho_{\text {ads }}$ : Densidad de la fase adsorbida en libras por pie cúbico $[L b / p c]$.

$\rho_{g(p, T)}$ : Es la densidad de la fase gaseosa en función de la $P$ y $T$ del sistema.

Este modelo presenta un factor de corrección adicional sobre el modelo de dos parámetros al tener en cuenta la razón entre las densidades de las fases total y adsorbida.

\subsubsection{Modelo de DubiniN*- RADUSHKEVICH (DR)}

Según Dada et al. (2012)[5], se aplica generalmente para los mecanismos de adsorción con distribuciones de energía Gausiana en superficies heterogéneas, y está dada por:

$$
\text { Vads }=V_{L} \exp \left[-D\left(\ln \frac{P 0}{P}\right)^{2}\right]
$$

Donde $\mathrm{P}_{0}$ es la presión de saturación [psi] y $D$ es una constante propia del modelo

\subsubsection{Modelo de TOTH}

La isoterma empírica propuesta por Toth en 1971 fue diseñada para tener en cuenta los efectos de la heterogeneidad de la superficie de adsorción tenidos en cuenta por sus parámetros.

$$
\text { Vads }=V_{L} \frac{p}{\left(b+P^{u}\right)^{1 / u}}
$$

Los parámetros $V_{L}$ (máxima cantidad adsorbida de Langmuir), $b$ y $u$ se deben ajustar para obtener resultados coherentes mediante el uso de valores adecuados para cada uno, aun cuando la presión tiende a valores extremos tales como cero o infinito. Para el primer caso $(\mathrm{p} \rightarrow 0)$, la ecuación se reduce a la ley de Henry, y para el segundo caso $(\mathrm{p} \rightarrow \infty)$, calcula un valor límite confiable [12].

\subsubsection{Modelo de Sips}

Según Loganathan, et al. (2015)[10] el modelo de Sips describe fuertemente la heterogeneidad de la superficie de adsorción (Es el caso de la matriz de la roca shale) y su ecuación es:

$$
\text { Vads }=V_{L} \frac{(b P)^{1 / a}}{1+(b P)^{1 / a}}
$$

Donde $a$ cuantifica la heterogeneidad de la superficie de adsorción, $V_{L}$ representa la capacidad máxima de adsorción, $b$ es la afinidad de adsorción, $V_{a d s}$ es la cantidad de sustancia adsorbida y $P$ la presión.

Esta ecuación alcanza un mejor ajuste a altas presiones ya que combina las ecuaciones de Freundlich y Langmuir, y ha sido aplicada como una "isoterma de Freundlich generalizada" para la ocupación multisitio por largas cadenas de hidrocarburos [13]. 


\subsection{MODELOS DE CUATRO PARÁMETROS}

\subsubsection{Modelo de Dubinin- AstaKHOV (DA)}

Según Rouquerol, et al. (2014) [12], Dubinin y Astakhov en 1970 propusieron una forma más generalizada de la ecuación de DR, que corrige ciertas deficiencias teniendo en cuenta un factor empírico entero $J$, el cual relaciona la distribución de tamaños de poros y varía entre 2 - 6 de acuerdo a Rouquerol, et al (2014) y entre 1 - 4 según Clarkson, et al. (1997) [4]. Esta ecuación ha sido aplicada a una gran variedad de sistemas de adsorción, incluyendo carbón activado.

$$
\text { Vads }=V_{L} \exp \left[-D\left(\ln \frac{P 0}{P}\right)^{J}\right]
$$

\subsubsection{MOdELO DE FritZ- SCHLUNDER (FS)}

El modelo de Fritz-Schlunder (1974) de cuatro parámetros es el siguiente:

$$
\text { Vads }=\frac{A P^{\propto}}{1+B P^{\beta}}
$$

Donde $A$ y $B$ son los parámetros de FS cuyas unidades son volumen de gas sobre masa de adsorbente, y $\alpha$ y $\beta$ son los exponentes de la ecuación de FS y se encuentran en el rango de cero a uno [8].

\subsection{MODELO DE CINCO PARÁMETROS}

\subsubsection{Modelo de Fritz- SCHLUNDER (FS)}

Fritz y Schlunder proponen un modelo de cinco parámetros dado por:

$$
\text { Vads }=\frac{q_{m F S S} K_{1} P^{m_{1}}}{1+K_{2} P^{m_{2}}}
$$

Donde $q_{m F S S}$ es la capacidad máxima de adsorción de FS, y $K_{1}, K_{2}, m_{1}$ y $m_{2}$ son los parámetros propios de este modelo [8].

\section{PARAMETRIZACION DE LOS MODELOS DE ADSORCION}

\subsection{DATOS EXPERIMENTALES}

Los datos experimentales se tomaron del trabajo realizado por Gasparik et al. (2012) [7], el cual consistió en pruebas de adsorción de metano llevadas a cabo en una muestra de shale perteneciente a la formación Posidonea ubicada en los Países Bajos a una profundidad de $2491 \mathrm{~m}$ ( $8173 \mathrm{ft}$ ), correspondiente a la era Mesozoica, con una composición en $(\% w / w)$ dada por: $9 \%$ contenido de cuarzo y feldespatos, $34 \%$ filosilicatos, $49 \%$ carbonatos y un \%TOC reportado de 7,91 los cuales se presentan en la Tabla 1.

Las condiciones finales para la prueba de adsorción de metano fueron $65^{\circ} \mathrm{C}(608,67 \mathrm{R})$ y presiones

\begin{tabular}{|c|c|c|}
\hline Densidad (Lb/pc) & Presión (psi) & Vads (pce/ton) \\
\hline 0,000 & 0,00 & 0,000 \\
\hline 0,269 & 108,78 & 12,602 \\
\hline 0,578 & 230,61 & 23,201 \\
\hline 0,880 & 349,54 & 32,047 \\
\hline 1,179 & 464,12 & 38,891 \\
\hline 1,470 & 574,35 & 44,399 \\
\hline 1,844 & 715,03 & 51,409 \\
\hline 2,208 & 849,92 & 56,667 \\
\hline 2,560 & 977,55 & 61,591 \\
\hline 2,902 & 1100,84 & 65,430 \\
\hline 3,231 & 1218,32 & 68,935 \\
\hline 3,699 & 1383,66 & 72,774 \\
\hline 4,145 & 1541,75 & 76,113 \\
\hline 4,569 & 1691,14 & 78,533 \\
\hline 4,970 & 1831,83 & 80,536 \\
\hline 5,350 & 1966,71 & 82,122 \\
\hline 5,905 & 2165,42 & 83,874 \\
\hline 6,404 & 2345,26 & 85,460 \\
\hline 6,850 & 2509,16 & 86,211 \\
\hline 7,243 & 2657,10 & 87,296 \\
\hline 7,587 & 2789,08 & 87,463 \\
\hline 7,922 & 2919,61 & 87,379 \\
\hline 8,191 & 3026,94 & 87,463 \\
\hline 8,409 & 3115,42 & 87,797 \\
\hline 8,586 & 3187,94 & 88,131 \\
\hline 8,729 & 3247,40 & 88,047 \\
\hline 8,836 & 3293,81 & 87,964 \\
\hline 8,930 & 3332,97 & 88,548 \\
\hline 9,007 & 3366,33 & 88,297 \\
\hline 9,069 & 3392,44 & 88,464 \\
\hline 9,119 & 3414,19 & 88,381 \\
\hline 9,159 & 3431,60 & 88,548 \\
\hline
\end{tabular}
manométricas de hasta $25 \mathrm{MPa}(3625,94 \mathrm{Psi})$.

Tabla 1. Datos Experimentales.

Fuente: GASPARIK, M., et al. (2012): Supporting information. 


\subsection{PROCESO DE AJUSTE}

A los datos de adsorción de metano se les realizó un ajuste no lineal, con ayuda de la herramienta Solver del software Excel, la cual minimiza la función del error porcentual promedio (APE por sus siglas en inglés) por medio de la variación de los parámetros ajustables de cada modelo.

$$
\begin{gathered}
A P E(\%)= \\
\frac{\sum_{i=1}^{N}\left|\left((n)_{\text {medido }}-(n)_{\text {modelo }}\right) /(n)_{\text {medido }}\right|}{N} * 100
\end{gathered}
$$

Donde $\mathrm{N}$ es el número de datos experimentales.

Este ajuste se realizó a cada uno de los modelos descritos con el fin de determinar cada uno de sus parámetros.

\subsection{RESULTAdOS}

En la Figura 1 se evidencian las isotermas de adsorción generadas por los modelos de dos parámetros de Freundlich, Temkin y Langmuir. Entre las ecuaciones de dos parámetros, la isoterma que presenta la mejor representación de los datos experimentales es la de Langmuir, la cual arroja un error porcentual promedio de 2,244 . De los tres modelos el que presenta el mayor porcentaje de error es la ecuación de Freundlich, ya que cuando la presión tiende a infinito no propone una limitante a la capacidad máxima de adsorción.

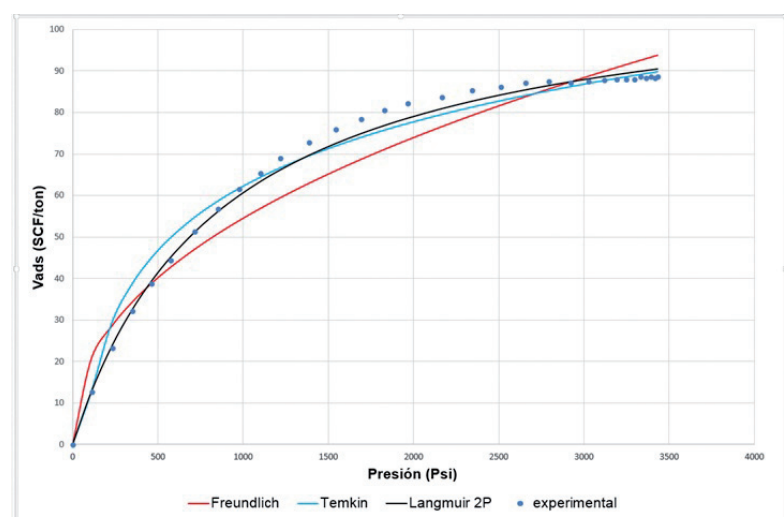

Figura 1. Modelos de dos parámetros

Los modelos de tres parámetros de Sips, Toth, Langmuir y DR empleados para la generación de las isotermas de adsorción se resumen en la Figura 2. De los cuatro modelos, el que presenta un mejor ajuste a los datos experimentales es el de Langmuir, el cual arroja un error porcentual promedio de 0,833; Sin embargo, los valores determinados para sus coeficientes no cumplen con la teoría de Langmuir, ya que la presión de Langmuir no coincide con la presión a la cual se ha adsorbido la mitad de la capacidad máxima de adsorción o volumen de Langmuir.

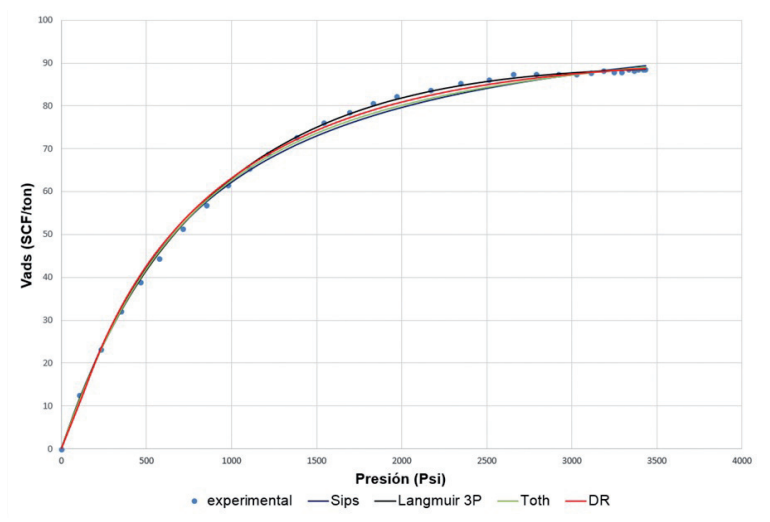

Figura 2. Modelos de tres parámetros

Los modelos de cuatro parámetros usados para la generación de la isoterma de adsorción son los de Fritz-Schlunder y Dubinin-Astakhov. En la Figura 3 se observa que el modelo de FS se ajusta mejor a los datos experimentales ya que su error porcentual promedio de 0,757 es menor que el presentado por el modelo de DA.

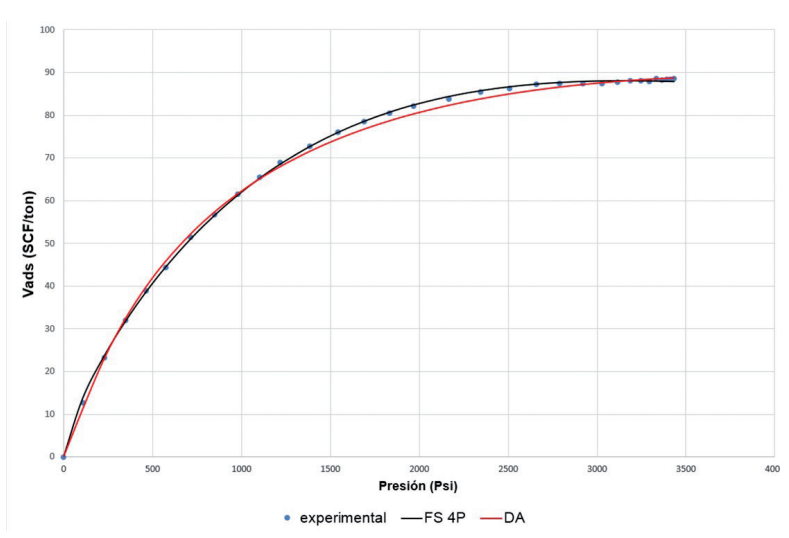

Figura 3. Modelos de cuatro parámetros

En la Figura 4 se observa la isoterma de adsorción generada por el modelo de Fritz-Schlunder de cinco parámetros. Entre los diez modelos estudiados, este es el que presenta el error porcentual promedio más bajo $(0,370)$. 


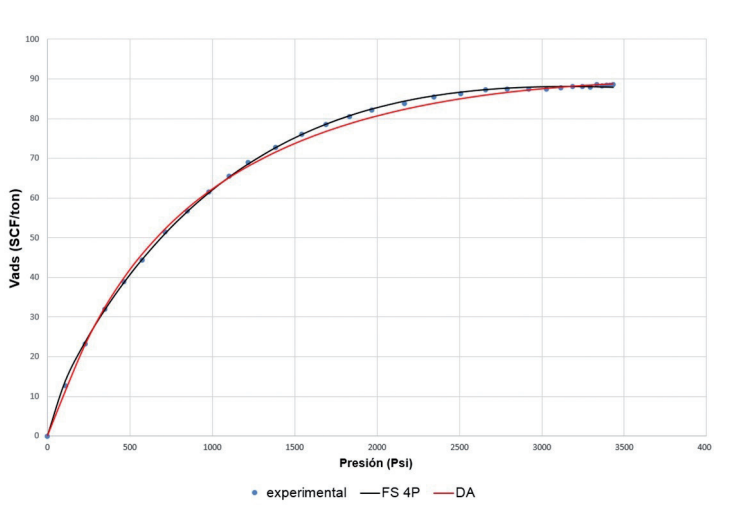

Figura 4. Modelo de cinco parámetros

Los modelos que mejor se ajustaron a los datos experimentales fueron los de mayor complejidad matemática, es decir, los que en sus ecuaciones proponen el mayor número de parámetros ya que a través de estos involucra más la fenomenología del proceso de adsorción.

En la Tabla 2 se resumen los parámetros de las isotermas de adsorción obtenidas mediante el ajuste no lineal y el respectivo error porcentual promedio de los diez modelos utilizados.

Tabla 2. Parametrización de las isotermas de adsorción

\begin{tabular}{|c|c|c|c|c|}
\hline \multirow{7}{*}{ 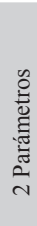 } & \multicolumn{3}{|c|}{ Modelo } & \multirow{3}{*}{$\begin{array}{l}\mathbf{A P E} \\
9,255\end{array}$} \\
\hline & \multirow{2}{*}{ Freundlich } & $k$ & 8,818203 & \\
\hline & & $m$ & 2,271909 & \\
\hline & \multirow{2}{*}{ Temkin } & $C$ & 22,36017 & \multirow{2}{*}{4,783} \\
\hline & & $A^{T}$ & 0,016152 & \\
\hline & \multirow{2}{*}{ Langmuir } & $V_{L}$ & 113,4259 & \multirow{2}{*}{2,244} \\
\hline & & $b$ & 0,001149 & \\
\hline \multirow{12}{*}{ 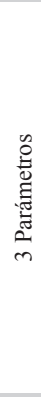 } & \multirow{3}{*}{ Sips } & $V_{L}$ & 104,8897 & \multirow{3}{*}{1,906} \\
\hline & & $b$ & 0,001403 & \\
\hline & & $m$ & 0,896473 & \\
\hline & \multirow{3}{*}{ DR } & $V_{L}$ & 89,92942 & \multirow{3}{*}{1,710} \\
\hline & & $D$ & 0,150959 & \\
\hline & & $P_{0}$ & 4612,52 & \\
\hline & \multirow{3}{*}{ Toth } & $V_{L}$ & 99,91005 & \multirow{3}{*}{1,594} \\
\hline & & $b$ & 0,001109 & \\
\hline & & $m$ & 1,342893 & \\
\hline & \multirow{3}{*}{ Langmuir } & $V_{L}$ & 153,4891 & \multirow{3}{*}{0,833} \\
\hline & & $P_{L}$ & 1291,58 & \\
\hline & & $\rho a d s$ & 44,41389 & \\
\hline \multirow{8}{*}{ 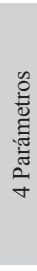 } & \multirow{4}{*}{ DA } & $V_{L}$ & 89,34050 & \multirow{4}{*}{1,386} \\
\hline & & $D$ & 0,198936 & \\
\hline & & $m$ & 1,825102 & \\
\hline & & $P_{0}$ & 3999,99 & \\
\hline & \multirow{4}{*}{ FS } & $A$ & 0,449069 & \multirow{4}{*}{0,757} \\
\hline & & $B$ & 0,000002 & \\
\hline & & $\alpha$ & 0,732173 & \\
\hline & & $\beta$ & 1,585381 & \\
\hline \multirow{5}{*}{ 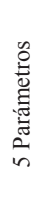 } & \multirow{5}{*}{ FS } & $q_{m F}$ & 96,84090 & \multirow{5}{*}{0,370} \\
\hline & & $K_{I}$ & 0,003002 & \\
\hline & & $m_{l}$ & 0,807595 & \\
\hline & & $K_{2}$ & 0,000013 & \\
\hline & & $m_{2}$ & 1,423499 & \\
\hline
\end{tabular}

\section{CÁlCULO DE LA CAPACIDAD TOTAL DE ALMACENAMIENTO DE GAS}

Una gran cantidad de metodologías han sido desarrolladas con el fin de calcular la Capacidad Total de Almacenamiento de Gas (CTAG) en formaciones de shale gas, las cuales van desde modelos sencillos que emplean la ecuación volumétrica hasta aquellos que requieren de datos de pruebas de presión en el estado transiente y uso de curvas tipo para realizar la estimación del gas en sitio [11].

\subsection{Metodología}

En el presente estudio se empleó la metodología propuesta por Ambrose, et al (2010)[3]; la cual surgió mediante la combinación de la ecuación volumétrica para el cálculo de gas libre con la isoterma de Langmuir de 2 parámetros que modela el comportamiento del gas adsorbido; obteniendo de este modo la ecuación que se presenta a continuación:

$$
\begin{gathered}
G_{s t}=32,0368 \frac{\emptyset\left(1-S_{w}\right)-1,318 \times 10^{-6} M \frac{\rho_{b}}{\rho_{s}}\left(\frac{V_{L} P}{P+P_{L}}\right)}{\rho_{b} B_{g}} \\
+\frac{V_{L} P}{P+P_{L}}
\end{gathered}
$$

En donde el primer término tiene en cuenta el volumen de gas libre corregido por la presencia de gas adsorbido y el segundo término representa el volumen de gas adsorbido. Además, $B_{g}$ representa el factor volumétrico de formación del gas [pc/pce], $M$ el peso molecular [Lb/ Lbmol], $\rho_{s}$ la densidad de la fase adsorbida $\left[\mathrm{g} / \mathrm{cm}^{3}\right], \rho_{b}$ la densidad de la roca $\left[\mathrm{g} / \mathrm{cm}^{3}\right]$ y $V_{L}$ [pce/ton] y $P_{L}[\mathrm{psi}]$, parámetros de adsorción de la isoterma de Langmuir, los cuales son dependientes de la composición del gas bajo investigación [9].

Los valores utilizados para cada parámetro necesario en el cálculo de la CTAG se presentan en la Tabla 3. Algunos de ellos fueron tomados del estudio realizado por Gasparik, et al. (2012)[7] a la formación Posidonea; sin embargo, fue necesario el uso de otros datos encontrados en la literatura con el fin de complementar la información requerida para poder realizar dicho cálculo. [1] 
Tabla 3. Parámetros necesarios para el cálculo del GOES

\begin{tabular}{|ccc|}
\hline Parámetro & Valor & Símbolo \\
\hline Porosidad, \% & 4,8 & $\varnothing$ \\
\hline Saturación de agua, \% & 35 & $S_{w}$ \\
\hline Saturación de aceite, $\%$ & 0 & $S_{o}$ \\
\hline Peso molecular del metano, $\mathrm{lb} / \mathrm{lb}-\mathrm{mol}$ & 16 & $M$ \\
\hline Densidad de la roca, $\mathrm{g} / \mathrm{cm}^{3}$ & 2,5 & $\rho_{b}$ \\
\hline Densidad de la fase adsorbida, g/cm ${ }^{3}$ & $0,371^{[9]}$ & $\rho_{s}$ \\
\hline Presión de yacimiento, psi & 3710 & $P$ \\
\hline Volumen de Langmuir, pce/ton & 113,43 & $V_{L}$ \\
\hline Presión de Langmuir, psi & 870,3 & $P_{L}$ \\
\hline Factor volumétrico de formación del & 0,00478 & $B_{g}$ \\
\hline gas, pc/pce & & \\
\hline
\end{tabular}

El gas libre $\left(G_{f}\right)$, el gas adsorbido $\left(G_{a}\right)$ y la capacidad total de almacenamiento $\left(G_{s t}\right)$ para la formación hipotética son respectivamente,

$G_{f}=\frac{32,0368}{B_{g}}\left[\frac{\varnothing\left(1-S_{w}\right)}{\rho_{b}}-\frac{1,318 \times 10^{-6} M}{\rho_{s}}\left(\frac{V_{L} P}{P+P_{L}}\right)\right]$

$=\frac{32,0368}{0,00478}\left[\frac{0,048(1-0,35)}{2,5}\right.$

$\left.-=\frac{1,318 \times 10^{-6} * 16}{0,371}\left(\frac{113,43 * 3710}{3710+870,3}\right)\right]$

$=48,63 \frac{p c e}{\text { ton }}$

$G_{a}=\frac{V_{L} P}{P+P_{L}}$

$=\frac{113,43 * 3710}{3710+870,3}=91,88 \mathrm{pce} /$ ton

$G_{s t}=G_{f}+G_{a}$

$G_{s t}=140,51 \mathrm{pce} / \mathrm{ton}$

Los cálculos realizados muestran una capacidad de almacenamiento total de gas de 140,51 pce/ton, del cual, el mayor contribuyente es el gas adsorbido con un valor de 91,88 pce/ton que equivale al $65,39 \%$ a las condiciones propuestas en el ejercicio.

Posteriormente, con el fin de mejorar la precisión del método de Ambrose et al (2010), se sustituyó la isoterma de Langmuir de 2 parámetros por los demás modelos estudiados anteriormente, y se procedió a recalcular la capacidad de almacenamiento total de gas para cada uno de ellos.

\subsection{MODIFICACIONES AL MÉTODO DE AMBrose ET AL. (2010)}

Las ecuaciones obtenidas al modificar el modelo con las diferentes isotermas de adsorción se presentan en el anexo A. Además, los cálculos de gas adsorbido $(\mathrm{Ga})$, gas libre (Gf) y gas total (Gst) obtenidos con cada una de ellas, se presentan en la Tabla 4 y en la Figura 5.

\subsection{RESULtAdos}

Tabla 4. Resultados de los modelos modificados de Ambrose et al. (2010)

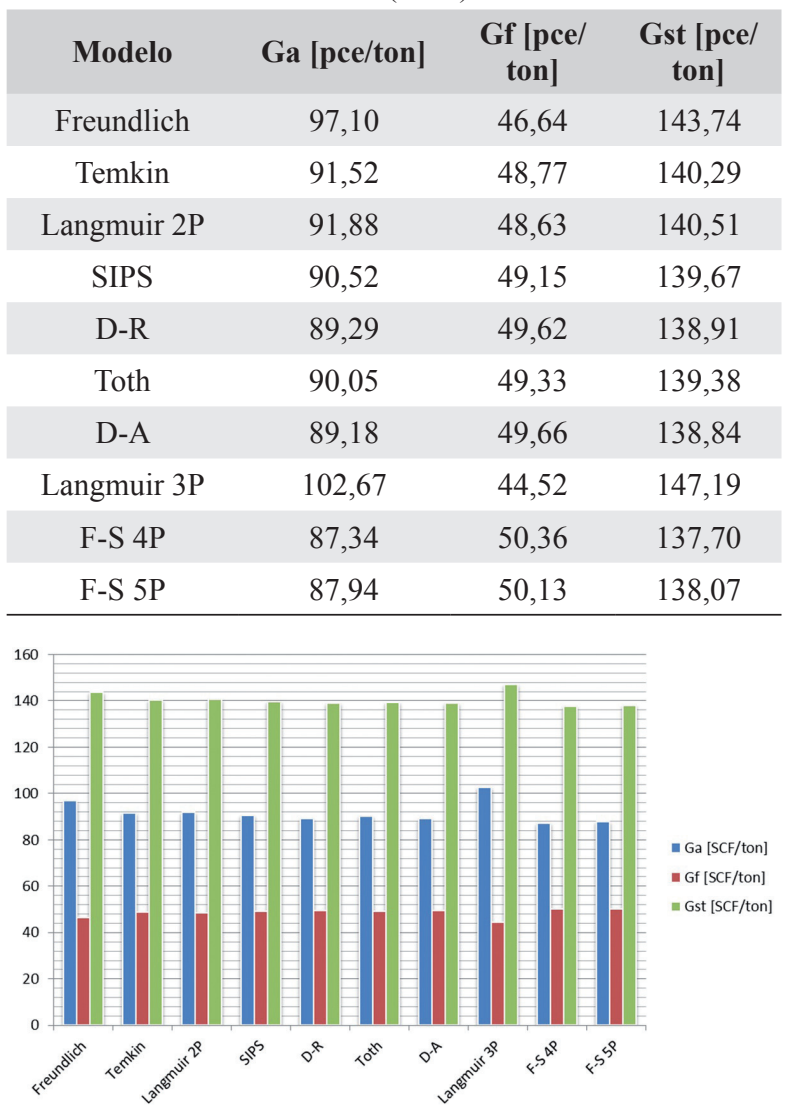

Figura 5. Resultados de los modelos modificados de Ambrose et al. (2010)

En la Figura 5 se evidencia que los modelos que se ajustaron mejor a los datos experimentales en la sección anterior y que se encuentran a la derecha de la gráfica, calcularon un menor volumen de gas adsorbido, mayor cantidad de gas libre y menor CTAG por tonelada de roca que las ecuaciones con mayor porcentaje de error. Esto se traduce en una menor cantidad de gas original en sitio.

Un caso particular observado fue el obtenido con el modelo de Langmuir de tres parámetros, el cual se 
desvió de la tendencia decreciente mostrada en la figura, debido a que los parámetros y con los cuales se ajustó el modelo no cumplen totalmente con las definiciones de volumen y presión de Langmuir.

\section{CONCLUSIONES}

La elección de un modelo representativo del comportamiento de la isoterma de adsorción es esencial para hacer predicciones y/o evaluaciones del potencial de los recursos en las formaciones shale.

Los modelos que mejor se ajustaron al comportamiento de los datos de adsorción experimentales estudiados, fueron los que en sus ecuaciones tenían mayor número de parámetros; siendo el de Fritz-Schlunder de cinco parámetros el que tuvo el error porcentual promedio más bajo.

El modelo de Freundlich sobrestimó la capacidad total de almacenamiento de gas de la formación, debido a su naturaleza empírica y falta de bases teóricas que la sustenten, tal como ha sido expresado por diferentes autores en sus respectivos estudios.

El mayor contribuyente a la CTAG bajo las condiciones establecidas es el gas adsorbido por la roca. Además, los modelos que estimaron mayor cantidad de gas adsorbido también calcularon mayor volumen de gas total en sitio. Por esta razón, es muy importante seleccionar de manera adecuada el modelo a emplear dada las implicaciones que representa.

\begin{tabular}{|c|c|}
\hline $\begin{array}{l}\text { Unidades } \\
\text { Empleadas }\end{array}$ & Unidades SI \\
\hline 1 pc (Pie Cúbico) & $0,0283168 \mathrm{~m}^{3}$ \\
\hline $1 \mathrm{Lb}$ & $0,453592 \mathrm{Kg}$ \\
\hline 1 Lb-mol & 453,59 moles \\
\hline 1 ton & $1000 \mathrm{Kg}$ \\
\hline $1 \mathrm{psi}$ & $6894,76 \mathrm{~Pa}$ \\
\hline \multicolumn{2}{|c|}{ kelvin $(k)=\frac{\text { Rankine }(R)-491,67}{1,8}+273,15$} \\
\hline
\end{tabular}

\section{BIBLIOGRAFÍA}

1. AGENCIA NACIONAL DE HIDROCARBUROS. CUENCA CATATUMBO: Integración Geológica de la Digitalización y Análisis de Núcleos. Colombia, 2012.
2. AKHTAR, Arshia; KHAN, Md. Dilwar Alam and NABI, S.A. Ion Exchange Kinetics and Equilibrium Studies of Metal Ions Onto a Hybrid Cation Exchanger: Polyaniline-Sn(Iv)Tungstophosphate. En: International Journal of Engineering Sciences \& Research Technology. January, 2015. vol. 4, no. 1, p. 89-98.

3. AMBROSE, Ray J., HARTMAN, Robert C., DIAZ CAMPOS, Mery, AKKUTLU, I. Yucel, SONDERGELD, Carl H. Shale Gas-in-Place Calculation Part I: New Pore-Scale Considerations. En: SPE Unconventional Gas Conference (23-25 February, 2010: Pittsburgh, Pennsylvania). SPE 131772, p. 219-229.

4. CLARKSON, C. R.; BUSTIN, R. M. and LEVY, J. H. Application of the Mono/Multilayer and Adsorption Potential Theories to Coal Methane Adsorption Isotherms at Elevated Temperature and Pressure. En: Carbon. 1997, vol. 35, no. 12, p. 16891705.

5. DADA, A.O., OLALEKAN, A. P., OLATUNYA, A. M., DADA, O. Langmuir, Freundlich, Temkin and Dubinin-Radushkevich Isotherms Studies of Equilibrium Sorption of Zn2+ Unto Phosphoric Acid Modified Rice Husk. En: Journal of Applied Chemistry. 2012, vol. 3, no. 1, p. 38-45.

6. ETMINAN, S. Reza, JAVADPOUR, Farzam, MAINI, Brij B., CHEN, Zhangxin. Measurement of gas storage processes in shale and of the molecular diffusion coefficient in kerogen. En: International Journal of Coal Geology. October, 2013. No. 123, p. 10-19.

7. GASPARIK, M., GHANIZADEH, A., BERTIER, P., GENSTERBLUM, Y., BOUW, S., and KROSS, Bernhard M. High-Pressure Methane Sorption Isotherms of Black Shales from The Netherlands. En: Energy and Fuels American Chemical Society. July 5, 2012, vol. 26, p. 4995-5004.

8. HAMDAOUI, Oualid, NAFFRECHOUX, Emmanuel. Modeling of Adsorption Isotherms of Phenol and Chlorophenols onto Granular Activated Carbon Part Ii. Models with More Than Two Parameters. En: Journal of Hazardous Materials. 2007, vol. 147, no. 1-2, p. 401-411.

9. HARTMAN, Robert C., AMBROSE, Ray J., AKKUTLU, I. Yucel, CLARKSON, C.R. Shale 
Gas-in-Place Calculation Part II: Multi-Component Gas Adsorption Effects En: SPE Unconventional Gas Conference (14-16 June, 2011: Woodlands, Texas). SPE 144097, p. 2-17.

10. LOGANATHAN, Sravanthi, TIKMANI, Mayur, GHOSHAL, Aloke Kumar. Pore-expanded MCM41 for $\mathrm{CO}_{2}$ adsorption: Expermiental and isotherm modeling studies. En: Chemical Engineering Journal. June, 2015, no. 280, p. 9-17.

11. MENGAL, Salman A. y WATTENBARGER, R. A. Accounting for Adsorbed Gas in Shale Gas Reservoirs. En: Spe Middle East Oil and Gas Show and Conference. (25-28, September, 2011: Manama, Bahrain). Texas A\&M University, 2011. p. 1-15.

12. ROUQUEROL，F. ROUQUEROL， J.; SING, K.S.W.; LLWELLYN, P. and MAURIN, G. Adsorption by Powders and Porous Solids: Principles, Methodology and Applications. 2nd Ed. Marseilles: Academic Press, 2014, p.1-626.

13. SIPS, Robert. On the Structure of a Catalyst Surface. En: The Journal of Chemical Physics, Volume 16, Issue 5, 1948, p.490-495.

14. SUBRAMANYAM, B. and ASHUTOSH, D. Adsorption Isotherm Modeling of Phenol onto Natural soils - Applicability of Various Isotherm Models. En: International Journal of Environmental Research. 2011, vol. 6, no. 1, p.265-276.

\section{ANEXOS}

Anexo A. Modelos modificados de Ambrose et al 2010.

a) Método Convencional (Langmuir 2 Párametros) de Ambrose et al 2010.

$$
\begin{gathered}
G_{s t}=32,0368 \frac{\emptyset\left(1-S_{w}\right)-1,318 \times 10^{-6} M \frac{\rho_{b}}{\rho_{s}}\left(\frac{V_{L} P}{P+P_{L}}\right)}{\rho_{b} B_{g}} \\
+\frac{V_{L} P}{P+P_{L}}
\end{gathered}
$$

b) Método Modificado de Ambrose (Freundlich).

$$
\begin{gathered}
G_{s t}=32,0368 \frac{\emptyset\left(1-S_{w}\right)-1,318 \times 10^{-6} M \frac{\rho_{b}}{\rho_{s}}\left((k P)^{1 / m}\right)}{\rho_{b} B_{g}} \\
+(k P)^{1 / m}
\end{gathered}
$$

c) Método Modificado de Ambrose (Temkin).

$$
\begin{gathered}
G_{s t}=32,0368 \frac{\emptyset\left(1-S_{w}\right)-1,318 \times 10^{-6} M \frac{\rho_{b}}{\rho_{s}}(C * \ln (A \tau P))}{\rho_{b} B_{g}} \\
+\operatorname{Cln}(A \tau P)
\end{gathered}
$$

d) Método Modificado de Ambrose (SIPS).

$$
\begin{gathered}
G_{s t}=32,0368 \frac{\emptyset\left(1-S_{w}\right)-1,318 \times 10^{-6} M \frac{\rho_{b}}{\rho_{s}}\left(V_{L} \frac{(b P)^{1 / m}}{1+(b P)^{1 / m}}\right)}{\rho_{b} B_{g}} \\
+V_{L} \frac{(b P)^{1 / m}}{1+(b P)^{1 / m}}
\end{gathered}
$$

e) Método Modificado de Ambrose (DubininRadushkevich).

$$
\begin{gathered}
G_{s t}=32,0368 \frac{\emptyset\left(1-S_{w}\right)-1,318 \times 10^{-6} M \frac{\rho_{b}}{\rho_{s}}\left(V_{L} \exp \left[-D\left(\ln \frac{P_{0}}{P}\right)^{2}\right]\right)}{\rho_{b} B_{g}} \\
+V_{L} \exp \left[-D\left(\ln \frac{P_{0}}{P}\right)^{2}\right]
\end{gathered}
$$

f) Método Modificado de Ambrose (Toth).

$$
\begin{gathered}
G_{s t}=32,0368 \frac{\emptyset\left(1-S_{w}\right)-1,318 \times 10^{-6} M \frac{\rho_{b}}{\rho_{s}}\left(V_{L} \frac{P}{\left(b+P^{m}\right)^{1 / m}}\right)}{\rho_{b} B_{g}} \\
+V_{L} \frac{P}{\left(b+P^{m}\right)^{1 / m}}
\end{gathered}
$$

g) Método Modificado de Ambrose (Dubinin-Astakhov).

$$
\begin{gathered}
G_{s t}=32,0368 \frac{\emptyset\left(1-S_{w}\right)-1,318 \times 10^{-6} M \frac{\rho_{b}}{\rho_{s}}\left(V_{L} \exp \left[-D\left(\ln \frac{P 0}{P}\right)^{m}\right]\right)}{\rho_{b} B_{g}} \\
+V_{L} \exp \left[-D\left(\ln \frac{P_{0}}{P}\right)^{m}\right]
\end{gathered}
$$


h) Método Modificado de Ambrose (Langmuir 3 Parámetros).

$$
\begin{gathered}
G_{s t}=32,0368 \frac{\emptyset\left(1-S_{w}\right)-1,318 \times 10^{-6} M \frac{\rho_{b}}{\rho_{s}}\left(V_{L} \frac{P}{P+P_{L}}\left(1-\frac{\rho_{g}(P, T)}{\rho_{a d s}}\right)\right)}{\rho_{b} B_{g}} \\
+V_{L} \frac{P}{P+P_{L}}\left(1-\frac{\rho_{g}(P, T)}{\rho_{a d s}}\right)
\end{gathered}
$$

i) Método Modificado de Ambrose (Fritz-Schlunder 4 Parámetros).

$$
\begin{gathered}
G_{s t}=32,0368 \frac{\emptyset\left(1-S_{w}\right)-1,318 \times 10^{-6} M \frac{\rho_{b}}{\rho_{s}}\left(\frac{A P^{\alpha}}{1+B P^{\beta}}\right)}{\rho_{b} B_{g}} \\
+\frac{A P^{\alpha}}{1+B P^{\beta}}
\end{gathered}
$$

j) Método Modificado de Ambrose (Fritz-Schlunder 5 Parámetros).

$$
\begin{gathered}
G_{s t}=32,0368 \frac{\emptyset\left(1-S_{w}\right)-1,318 \times 10^{-6} M \frac{\rho_{b}}{\rho_{s}}\left(\frac{q_{m F S S} K_{1} P^{m_{1}}}{1+K_{2} P^{m_{2}}}\right)}{\rho_{b} B_{g}} \\
+\frac{q_{m F S S K} K^{m_{1}}}{1+K_{2} P^{m_{2}}}
\end{gathered}
$$

Recepción: 5 de Agosto de 2015

Aceptación: 18 de Octubre de 2015 